\title{
Modelamiento Computacional de la Dinamica de Transmisión de la Varicela mediante Automatas Celulares (Cell-DEVS)

\author{
Neisser Pino Romero, ${ }^{1}$ Roxana López Cruz ${ }^{3}$ y Gabriel Wainer ${ }^{2}$
}

Resumen: En el presente trabajo, se realiza un modelo computacional mediante los Autómatas Celulares (Cell-DEVS) que describa la dinámica de transmisión de la Varicela en un grupo cerrado de personas donde se pueda propagar la enfermedad. Desde la perspectiva de la epidemiología matemática se tiene el modelo matemático SEIR de W. O. Kermack y A. G. McKendrick que representa la dinámica de la epidemia, en nuestro caso la Varicela, donde se realizará las simulaciones computacionales tanto por los Métodos Numéricos como los Autómatas Celulares para analizar el desarrollo de la enfermedad.

Palabras clave: epidemiología matemática; ecuaciones diferenciales; autómatas celulares; formalismo DEVS; simulaciones computacionales.

\section{Computational Modeling of Varicella Transmission Dynamics through Cellular Automata (Cell-DEVS)}

\begin{abstract}
In the present paper, a computational model is performed by Cellular Automata (Cell-DEVS) that describes the transmission dynamics of Varicella in a closed group of people where the disease can be spread. From the perspective of mathematical epidemiology we have the mathematical model SEIR of W.O. Kermack and A.G. McKendrick that represent the dynamics of the epidemic, in our case Varicela, where the computational simulations are performed both by the Numerical Methods and the Cellular Automata to analyze the development of the disease.
\end{abstract}

Keywords: mathematical epidemiology; differential equations; cellular automata; DEVS formalism; computational simulations.

Recibido: 02/09/2017. Aceptado: 26/12/2017. Publicado online: 31/12/2017

(CLos autores. Este artículo es publicado por la Revista PESQUIMAT de la Facultad de Ciencias Matemáticas, Universidad Nacional Mayor de San Marcos. Este es un artículo de acceso abierto, distribuido bajo los términos de la licencia Creative Commons Atribucion-No Comercia-Compartir Igual 4.0 Internacional.(http://creativecommons.org/licenses/by-nc-sa/4.0/) que permite el uso no comercial, distribución y reproducción en cualquier medio, siempre que la obra original sea debidamente citada. Para información, por favor póngase en contacto con revistapesquimat.matematica@unmsm.edu.pe

\footnotetext{
${ }^{1}$ Universidad Peruana Cayetano Heredia, e-mail: neisser.pino@upch.pe

${ }^{3}$ UNMSM, Facultad de Ciencias Matemáticas, e-mail: rlopezc@unmsm.edu.pe

${ }^{2}$ Carleton University, Department of Systems and Computer Engineering, e-mail: gwainer@sce.carleton.ca
} 


\section{Introducción}

En 1927, W. O. Kermack y A. G. McKendrick propusieron un modelo matemático que intentaba describir el comportamiento de las enfermedades infecciosas, en nuestro caso será la Varicela, en una primera consideración se tuvo las Ecuaciones Diferenciales Ordinarias, en su tiempo fue un gran aporte que realizó la matemática en cuanto al estudio de las epidemias. Desde ahí, se comenzó a recurrir al modelamiento matemático para intentar describir diferentes fenómenos en el área de la salud. Del mismo modo, se realizó un modelamiento mediante las Ecuaciones Diferenciales Parciales, donde se considera el tiempo-espacio que podría describir la difusión de la epidemia a través del contacto espacial mediante el tiempo de considerado. Pero los diferentes fenómenos no resultaban sencillos formalizarlos mediante los modelos matemáticos, en cuanto al problema que contenía más variables independientes a considerar. [1, 10, 12 y 16].

Desde ahí, la computación comenzó a abordar enormemente los problemas para poder describir problemas que representados mediante un sistema de ecuaciones es complicado y de difícil análisis tanto analítico como computacional. Por lo cual, se ha desarrollado los métodos numéricos para realizar las simulaciones computacionales, pero de similar manera se ha ido desarrollando en los últimos años otra perspectiva de realizar simulaciones computacionales, las cuales es abarcada por los Autómatas Celulares donde permiten representar de manera más sencilla muchos sistemas dinámicos de una gran complejidad. [13, 16 y 17].

\section{La Varicela}

Según la Dirección General de Epidemiologia (D.G.E), la varicela es una enfermedad infecciosa viral, muy contagiosa, que afecta principalmente a los niños. Los síntomas de esta enfermedad principalmente son fiebre y lesiones en la piel, tipo ampollas que producen mucha picazón, y que luego se hacen costra. Esta enfermedad se propaga fácilmente a través del aire cuando una persona que la tiene tose o estornuda; también se puede contagiar al tocar las ampollas de una persona infectada. El contagio se puede dar uno a dos días antes de que la persona presente el sarpullido (lesiones en la piel) hasta que todas las ampollas hayan formado costra. La varicela por lo general es leve en los niños, pero en algunos casos se puede complicar especialmente en menores de 5 años, adolescentes, adultos y personas que tiene las defensas bajas. Siendo una enfermedad que dura de 10 a 21 días y que usualmente se autolimita; en la mayor parte de los casos el tratamiento es reposo, fluidos y manejo de la fiebre. [8, 9 y 11].

\section{Modelo Matemático SEIR}

El modelo matemático SEIR aplicado a la dinámica de transmisión de la Varicela se puede expresar mediante dos tipos de Ecuaciones Diferenciales. En primer lugar, las Ecuaciones Diferenciales Ordinarias (E.D.O.) donde la variable independiente es el tiempo que describirá el desarrollo de la propagación de la enfermedad. En segundo lugar, las Ecuaciones Diferenciales Parciales (E.D.P.) contribuyen otra perspectiva de la propagación de la enfermedad donde las posee dos variables independientes cuales son el el tiempo y el espacio. [4, 12 y 13].

\subsection{Modelo Matemático SEIR en E.D.O.}

La población fundamental para describir la Varicela es la población de los Expuestos, debido que considera el periodo latente de la enfermedad, es decir, poseen la enfermedad pero aun no presentan los síntomas de la enfermedad. Después de este periodo de incubación pasan a la 
población de los Infectados, aptos para poder contagiar a los Susceptibles. Y, después del periodo considerado que la enfermedad en promedio afecta a los infectados, estos pasan a la población de los recuperados donde ya poseen la inmunidad ante la enfermedad, es decir, ya no pueden padecer de nuevo la enfermedad. El presente modelo considera que no existe natalidad ni mortalidad, es decir, la población total del modelo es constante en todo el tiempo considerado. Por lo cual, ahora formularemos el modelo matemático para comprender la dinámica de transmisión de la varicela, lo cual considerará las variables y los parámetros del modelo, el cual estará representado mediante un Sistema de Ecuaciones Diferenciales Ordinarias. [4, 5, 6 y 7].

Las variables consideradas (poblaciones epidemiológicas) son las siguientes:

- $S(t)$ : Población de los Susceptibles en el tiempo $t$.

- $E(t)$ : Población de los Expuestos en el tiempo $t$.

- $I(t)$ : Población de los Infectados en el tiempo $t$.

- $R(t)$ : Población de los Recuperados en el tiempo $t$.

Los parámetros considerados (tasas epidemiológicas) son las siguientes:

- $\beta$ : Tasa promedio de Contagio.

- $\phi$ : Tasa promedio de Incubación.

- $\gamma$ : Tasa promedio de Recuperación.

Teniendo las presentes consideraciones, el modelo matemático en E.D.O. estaría representado de la siguiente manera:

$$
\begin{aligned}
& S^{\prime}=-\beta \cdot S I \quad S(0)=S_{o} \geq 0 \\
& E^{\prime}=\beta \cdot S I-\phi \cdot E \quad E(0)=E_{o} \geq 0 \\
& I^{\prime}=\phi \cdot E-\gamma \cdot I \quad I(0)=I_{o} \geq 0 \\
& R^{\prime}=\gamma \cdot I \quad R(0)=R_{o} \geq 0
\end{aligned}
$$

El modelo matemático expresado en (1), representa la dinámica de la transmisión de la Varicela de acuerdo las consideraciones que se ha tenido para su formulación de acuerdo a la concepción que se tiene de la enfermedad en la sección 2. Por otro lado, la rigurosidad y la formalidad de la matemática exige que el sistema (1) esté bien definido para que satisfaga la Existencia y la Unicidad de las Soluciones, y además la estabilidad del modelo que se busca en todo el modelamiento matemático. Estos conceptos no se considerará como parte del análisis del presente trabajo. [1, 4, 6 y 13$]$.

\subsubsection{Simulación Computacional}

Las simulaciones computacionales nos permiten visualizar el comportamiento que realiza las soluciones, mediante los Métodos Numéricos que nos brinda soluciones aproximadas, en concreto, los problemas que contienen un Valor Inicial. Uno de los métodos más conocidos y utilizados es el método de Runge-Kutta de orden cuatro. Por lo cual, consideraremos las siguientes poblacines epidemiológicas iniciales, y también las tasas epidemiológicas correspondientes. Se considerará un tiempo de simulación de 30 días. Y el software utilizado para la simulación computacional será el MATLAB con el algoritmo computacional de Runge-Kutta. [3, 4 y 13].

$$
\begin{array}{|l|l|}
\hline S(0)=95 & I(0)=5 \\
\hline E(0)=0 & R(0)=0 \\
\hline
\end{array} \quad \begin{array}{|l|l|}
\hline \beta=0,50 & \gamma=0,20 \\
\hline \phi=0,40 & \multicolumn{1}{|c}{} \\
\hline
\end{array}
$$




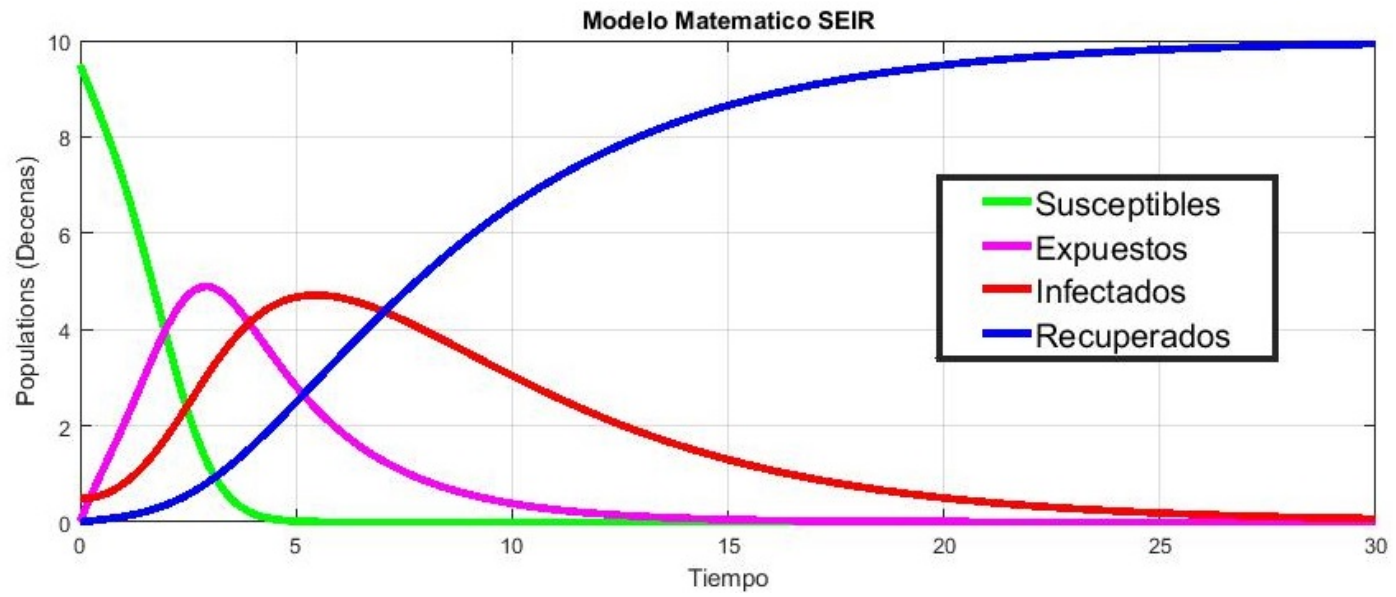

\subsubsection{Interpretación Epidemiológica}

La dinámica del comportamiento de las poblaciones epidemiológicas descrita por la gráfica indica como va evolucionando a través del tiempo, considera un rápido descenso de los Susceptibles, por mientras que el crecimiento acelerado de los Expuestos, que van experimentando el periodo latente de la enfermedad para lugar pasar a la población Infectada, aptos para propagar la enfermedad de manera directa mediante sus formas de contagio, las poblaciones de los Expuestos y de los Infectados tienen sus respectivos umbrales de crecimiento para luego empezar a descender. La población de los Recuperados en el tiempo va en aumento hasta llegar a obtener toda la población total, debido que se ha considerado que la población total es constante en todo el tiempo considerado (no existe natalidad ni mortalidad). [1, 9 y 11$]$.

El comportamiento de las poblaciones Infectadas es el centro del estudio en cuánto se puede propagar y cuánto puede durar la epidemia, considerando que la población de los Expuestos mientras se encuentran en su periodo latente no se puede determinar si están contagiados. Pero ante la aparición de un solo infectado se puede pasar a un estado de alerta epidemiológica que permita evitar más contagios en la población Suscetibles. Cabe recordar que se está considerando que después de contraer la enfermedad, y realizar el adecuado tratamiento indicado por la D.G.E se pasa a un estado de Recuperado donde no se puede contraer de nuevo la enfermedad, por lo cual se epidemiológicamente se considera inmune a la enfermedad. [9, 11 y 12].

\subsection{Modelo Matemático SEIR en E.D.P.}

Como ya se ha visto en la sección anterior en el Modelo SEIR desarrollado mediante las Ecuaciones Diferenciales Ordinarias, donde nuestra variable independiente es el tiempo. Ahora, se construirá el modelo SEIR desarrollado mediante las Ecuaciones Diferenciales Parciales donde las variables independientes son el tiempo y el espacio. Como se había mencionado el virus necesita algún tiempo para desarrollarse y volverse más fuerte. Durante este tiempo, la persona infectada no es contagiosa hasta que termine su período latente. Por lo cual, hacemos las siguientes suposiciones para la construcción del Modelo matemático, de una manera similar como se hizo anteriormente. [2, 9, 10 y 15$]$.

1. El modelo SEIR representa la dinámica de la infección en una población de tamaño $N$ donde no incluye la posición espacial (donde no se considera la tasa de mortalidad y la tasa natalidad).

2. $N(t, x)=S(t, x)+E(t, x)+I(t, x)+R(t, x)$ constante. 
3. Tenemos una mezcla homogénea, es decir, los individuos de la población hacen contacto en al azar y no se mezclan en su mayoría en un subgrupo más pequeño.

4. El modelo supone que los individuos recuperados son inmunes a la infección.

5. Los individuos infectados se trasladan a la población de los Expuestos (E), y después de un período latente promedio donde luego pasarán luego a la población de los Infectados.

6. Suponemos que la movilidad espacial se rige por coeficientes de difusión aleatoria, $D_{S}, D_{E}, D_{I}$ y $D_{R}$ para las clases Susceptibles, Expuestos, Infecciosas y Recuperados, respectivamente.

A todos los individuos no se les permite escapar o abandonar el entorno estudiado, es decir, para este modelo tenemos condiciones de frontera de Neumann homogéneas que representan un ambiente cerrado: $S_{x}(t, 0)=S_{x}(t, 1)=E_{x}(t, 0)=E_{x}(t, 1)=I_{x}(t, 0)=R_{x}(t, 1)=0$, con las condiciones iniciales: $S(0)=S_{o} \geq 0, E(0)=E_{o} \geq 0 ; I(0)=I_{o} \geq 0, \quad R(0)=R_{o} \geq 0$.

Bajo las siguientes consideraciones se tiene el modelo epidemiológico:

- $\beta$ : $\quad$ Tasa de Transmisión o de contagio.

- $\nu$ : Tasa de transisión de los Expuestos a los Infectados (período latente).

- $\tau$ : Tasa de Recuperación de la enfermedad.

- $D_{S}, D_{E}, D_{I}, D_{R}$ : Tasa de Difusión o Expansión

Donde las poblaciones epidemiológicas son las siguientes:

- $S(t, x)$ : Población de los Susceptibles en el tiempo $t$ y en el espacio $x$.

- $E(t, x): \quad$ Población de los Expuestos en el tiempo $t$ y en el espacio $x$.

- $I(t, x)$ : Población de los Infectados en el tiempo $t$ y en el espacio $x$.

- $R(t, x)$ : Población de los Recuperados en el tiempo $t$ y en el espacio $x$.

Por lo cual, el modelo matemático en E.D.P. estaría representado de la siguiente manera:

$$
\begin{aligned}
\frac{d S(t, x)}{d t} & =-\beta S(x, t) \cdot I(t, x)+D_{S} S_{x x}(t, x) \\
\frac{d E(t, x)}{d t} & =\beta S(x, t) \cdot I(t, x)-\nu E(t, x)+D_{E} E_{x x}(t, x) \\
\frac{d I(t, x)}{d t} & =\nu E(t, x)-\tau I(t, x)+D_{I} I_{x x}(t, x) \\
\frac{d R(t, x)}{d t} & =\tau I(t, x)+D_{R} R_{x x}(t, x)
\end{aligned}
$$

Del modelo matemático expresado en la ecuación (2), se puede tener una mejor concepción de la difusión de la enfermedad mediante el tiempo y el espacio considerado. Lo cual, puede representar mejor la dinámica de la transmisión de la enfermedad en el espacio-tiempo. El presente modelo matemático no se realizará la simulación computacional respectiva, debido que se enfocará en la simulación computacional del modelo matemático en E.D.O. y mediante de los Autómatas Celulares que se desarrollará en la siguiente sección. [7, 10, 12 y 15]. 


\section{Formalismo DEVS}

Los Autómatas Celulares (A.C.) surgen en la década de 1940 con John Von Neumann, el cual intentaba modelar una máquina que fuera capaz de autoreplicarse, llegando así a un modelo matemático de dicha máquina con reglas complicadas sobre una red rectangular. Por lo cual, un autómata celular es un modelo matemático para un sistema dinámico, conformado por un conjunto de celdas o células que adquieran distintos estados o valores. Estos estados son alterados de un instante a otro en unidades de tiempo discreto, es decir, que se puede cuantificar con valores enteros a intervalos regulares. [14, 16 y 17$]$.

Según Wainer y Giambiasi en [16] y Wainer [17] , el formalismo Cell-DEVS fue presentado como la combinación de los Autómatas Celulares (A.C.) y el formalismo DEVS, cada célula está definida como un Modelo atómico, y se determina el procedimiento para acoplar las células, es decir, se define la interacción de las células entre sí. Se ha mencionado bastante son la teoría computacional de los Autómatas Celulares en su manera abstracta. Por lo cual, ahora se definirá el formalismo Cell-DEVS para poder representar un problema que se está modelando mediante los Autómatas Celulares. Como se había desarrollado en la sección anterior, el problema de la dinámica de transmisión de la varicela en un sistema cerrado donde no hay entradas ni salidas (epidemiológicamente significa que es un entorno cerrado donde no hay natalidad ni mortalidad de personas). [9, 12, 16 y 17$]$.

Las consideraciones fundamentales de los Autómatas Celulares que se tomará en cuenta cuando realicemos la formalización en Cell-DEVS del modelo SEIR son las siguientes:

- Una celda estará activa, mientras se reciben eventos externos o se programan eventos internos.

- Una célula estará pasiva, cuando no hay más eventos programados para ser transmitidos.

- Cuando llega un evento (por ejemplo, porque un vecino ha cambiado de estado), se ejecuta la función de transición externa $\delta_{\text {ext }}$, y se activa la función $\tau$.

- Si el estado de la célula no cambia, la célula pasiva permanece en un estado inactivo. Si hay un cambio, la función de transición externa programa una transición interna después de un retardo.

Una vez que definimos el comportamiento de una sola célula, necesitamos formar un espacio celular. Incluimos la definición de los modelos acoplados a dos dimensiones de Cell-DEVS con vecinos adyacentes. La técnica de modelado mediante los Autómatas Celulares permite mantener la capacidad para describir sistemas complejos utilizando reglas muy sencillas, al mismo tiempo que nos permite cerrar la brecha entre un Tiempo Discreto y una descripción de Evento Discreto como DEVS. El uso de DEVS como mecanismo formal de especificación básica nos permite definir interacciones con modelos definidos en otros formalismos que se puedan definir. $[4,16,17]$.

\subsection{Formalización DEVS del Modelo SEIR}

Después de haber realizado una pequeña introducción al Formalismo DEVS para poder realizar el respectivo análisis mediante el modelo representado en Cell-DEVS. Luego de realizar la formalización, se definirá las reglas correspondientes para poder generar la simulación computacional desde la perspectiva de los Autómatas Celulares mediante el formalismo DEVS. El software que se utilizará, es el CD++ para realizar la Simulación Computacional. [16 y 17]. 
Por lo cual, en primer lugar realizaremos el formalismo del Modelo SEIR, y luego su respectiva simulación computacional.

$$
\text { Epidemia_SEIR }=<X, Y, D,\left\{M_{i}\right\},\left\{I_{i}\right\},\left\{Z_{i}\right\} \text {, select }>
$$

Donde:

- $X=\varnothing \quad Y=\varnothing$

- $D=\{$ cell $\}$

- $M_{\text {cell }}=$ Cell $-S E I R$

- $I_{\text {cell }}=\varnothing \quad Z_{i}=\varnothing$

- select $=($ Cell $-S E I R)$

$$
\text { Cell }-S E I R=<X_{\text {list }}, Y_{\text {list }}, I, X, Y, n,\left\{t_{1}, t_{2}, \cdots, t_{n}\right\}, \eta, N, C, B, Z, \text { select }>
$$

Donde:

- $X_{\text {list }}=\varnothing \quad Y_{\text {list }}=\varnothing$

- $I=<p^{x}, p^{y}>$ donde $: p^{x}=p^{y}=\varnothing$

- $X=\{0,1,2,3\}$

- $n=2 \quad \eta=2$

- $t_{1}=t_{2}=10$

- $N=\{(-1,-1),(-1,0),(-1,1),(0,-1),(0,0),(0,1),(1,-1),(1,0),(1,1)\}$

- $B=\varnothing$

- $Z=\left\{P_{i, j} Y_{1} \rightarrow P_{i, j-1} X_{1}, P_{i, j} Y_{2} \rightarrow P_{i+1, j} X_{2}, P_{i, j} Y_{3} \rightarrow P_{i, j+1} X_{3}, P_{i, j} Y_{4} \rightarrow P_{i-1, j} X_{4}, P_{i, j} Y_{5} \rightarrow\right.$ $P_{i, j} X_{5}, P_{i, j+1} Y_{1} \rightarrow P_{i, j} X_{1}, P_{i-1, j} Y_{2} \rightarrow P_{i, j} X_{2}, P_{i, j-1} Y_{3} \rightarrow P_{i, j} X_{3}, P_{i+1, j} Y_{4} \rightarrow P_{i, j} X_{4}, P_{i, j} Y_{5} \rightarrow$ $\left.P_{i, j} X_{5}\right\}$

$$
C_{i, j}=<I, X, S, Y, N, \delta_{i n t}, \delta_{e x t}, d, \tau, \lambda>
$$

Donde:

- $p^{x}=\left\{X_{k} \in\{0,1,2,3\}\right.$ donde $\left.k=\overline{1,9}\right\}$

- $p^{y}=\left\{Y_{k} \in\{0,1,2,3\}\right.$ donde $\left.k=\overline{1,9}\right\}$

- $X=\{0,1,2,3\} \quad Y=\{0,1,2,3\}$

- $N=\{(-1,-1),(-1,0),(-1,1),(0,-1),(0,0),(0,1),(1,-1),(1,0),(1,1)\}$

- $d=$ Tiempo (dias)

- $S X$ : Variables Descriptivas

$$
S X=\left\{\begin{array}{lll}
0 & : & \text { Celda de Persona Susceptible } \\
1 & : & \text { Celda de Persona Expuesta } \\
2 & : & \text { Celda de Persona Infectada } \\
3 & : & \text { Celda de Persona Recuperada }
\end{array}\right.
$$




\subsection{Reglas}

Las reglas que se definirán para representar la dinámica de transición de los diferentes estados de las células (en nuestro caso, representan estado de personas y la transmisión de la enfermedad) son las siguientes donde se considera el proceso de contagio, el periodo latente de la enfermedad y de recuperación de las mismas. [16].

\begin{tabular}{|c|c|c|}
\hline Reglas & Resultado & Estado de la Vecindad \\
\hline 1 & 1 & $(0,0)=0 \wedge \quad \#(2) \geq 1$ \\
\hline 2 & 2 & $(0,0)=1 \wedge$ PeriodoLatente \\
\hline 3 & 3 & $(0,0)=2 \wedge$ TiempoRecuperacion \\
\hline 4 & 3 & $(0,0)=3 \wedge$ TiempoInmunidad \\
\hline
\end{tabular}

\subsubsection{Colores de las Células}

$$
S X=\left\{\begin{array}{lll}
0 & : & \text { Celda de Persona Susceptible (Verde) } \\
1 & : & \text { Celda de Persona Expuesta (Rosado) } \\
2 & : & \text { Celda de Persona Infectada (Rojo) } \\
3 & : & \text { Celda de Persona Recuperada (Azul) }
\end{array}\right.
$$

\subsection{Simulación Computacional}

Del mismo modo, consideraremos las mismas poblaciones iniciales para poder realizar la respectiva simulación computacional. Con respecto al software, CD++ es un programa destinado a la modelización y a la simulación de eventos discretos cuales se organizan según el formalismo DEVS. CD ++ puede funcionar en diferentes modos: en modo autónomo (soló un ordenador), en modo servidor, en modo tiempo real y en modo paralelo con un cluster Linux. CD ++ se utiliza en línea de comandos. [14, 16 y 17].

\begin{tabular}{|c|c|}
\hline Población de Susceptible & $S(0)=95$ \\
\hline Población de Expuestos & $E(0)=0$ \\
\hline Población de Infectados & $I(0)=5$ \\
\hline Población de Recuperados & $R(0)=0$ \\
\hline
\end{tabular}

Hay que notar, que se definirá una malla cuadrangular de $10 \times 10$, con el objetivo que la malla pueda contener el estado de 100 células, las cuales representan a las poblaciones epidemiológicas, y teniendo que se tiene un sistema cerrado (no entrada ni salida de nuevas células) nos permite tener en cuenta el cambio de estado de las células de toda la población considerada.

\subsubsection{Consideraciones de las Reglas}

Las reglas consideradas en la subsección 4.2, delimita la información que se tiene sobre la enfermedad de manera especializada, es decir, la información que se tiene sobre el modo de contagio, el periodo latente y el tiempo de recuperación. [16 y 17].

- Periodo latente : 2 días (comúnmente)

- Tiempo Promedio de la Enfermedad : 15 días (10 días a 21 días)

- Tiempo de Recuperación : Siempre (Recuperados poseen Inmunidad) 


\subsubsection{Simmulación Computacional}

Ahora se realizará la respectiva simulación computacional, partiendo de las mismas poblaciones iniciales consideradas en la simulación anterior mediante los métodos numéricos. La simulación mediante los Autómatas Celulares nos permite visualizar como se propaga la epidemia considerando el tiempo y el espacio. [9, 12, 16 y 17$]$.

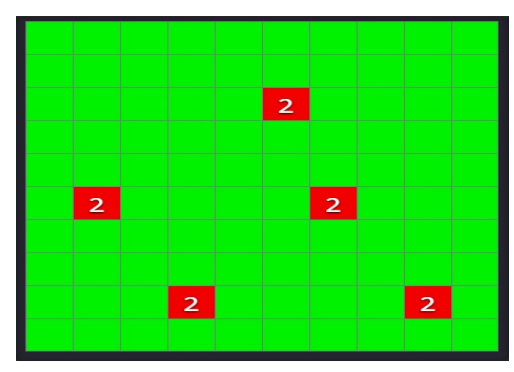

Teniendo la consideración de la posición permitiría predecir en que lugares la enfermedad puede propagarse más rápido o con menos rapidez. Este detalle es una gran ventaja cuando se considera un modelo computacional tiempo-espacio. Por lo cual, se presenta como evoluciona en el tiempo la enfermedad causando una epidemia que llega a su umbral, para luego descender y terminar ante la recuperación de todos individuos lo cual debe reflejarse en el modelo computacional para poder complementarlo adecuadamente. De aquí, se podría considerar que los modelos determínisticos, en nuestro caso por las Ecuaciones Diferenciales, y de una manera similar mediante los Autómatas Celulares, en nuestro por el formalismo DEVS (Cell-DEVS), pueden brindar mejores resultados para una interpretación epidemiológica más cercana a la realidad que se está estudiando. [16].

Después de realizar la simulación computacional mediante los Autómatas Celualres, se podrá generar una secuencia de estados mediante el tiempo considerado para la simulación que permitirá observar la evolución de la propagación de la enfermedad.

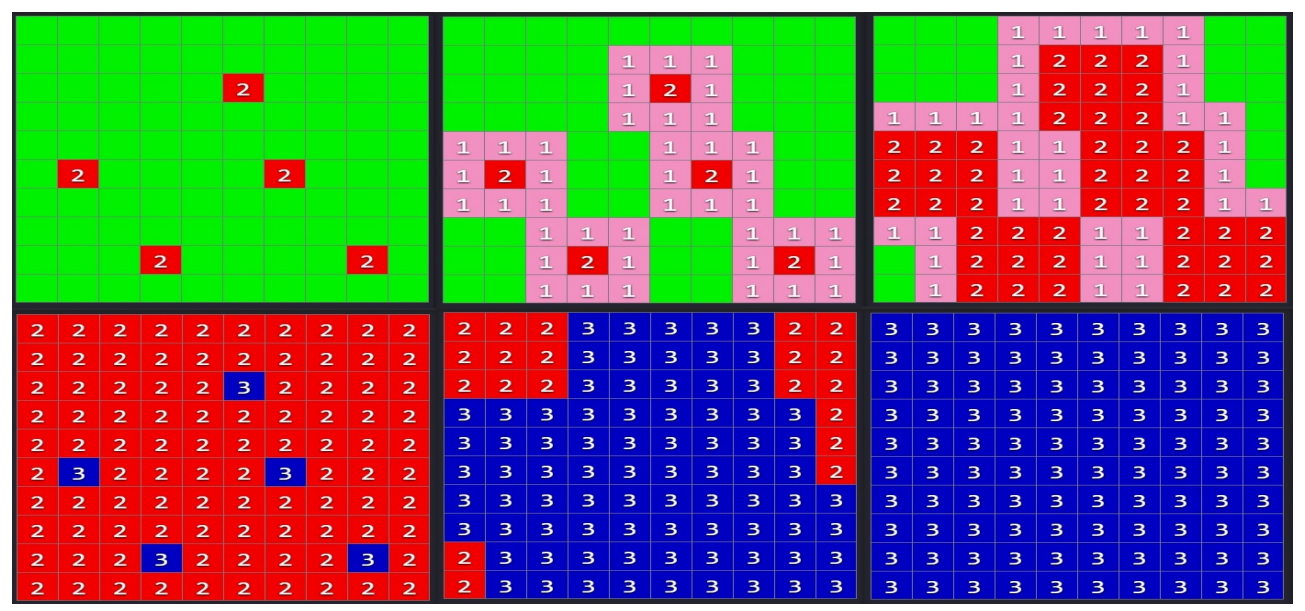

Cabe resaltar, el estado final de la simulación computacional donde se puede apreciar que la población total pertenece a la población de los Recuperados. Esto nos permite considerar que la simulación mediante los autómatas celulares complementa de una manera detallada de como se propaga la enfermedad, y el periodo latente de la enfermedad se puede observar como va abarcando toda la población, pero con el tiempo el periodo latente pasa a su periodo activo, es decir, los Expuestos pasaron a considerarse Infectados, aptos para propagar la enfermedad. Desde 
ahí, la epidemia va en descenso mientras se genera la recuperación de la población infectada. Desde aquí, se puede construir las curvas de evolución de las poblaciones epidemiológicas. [9, 16 y 17].

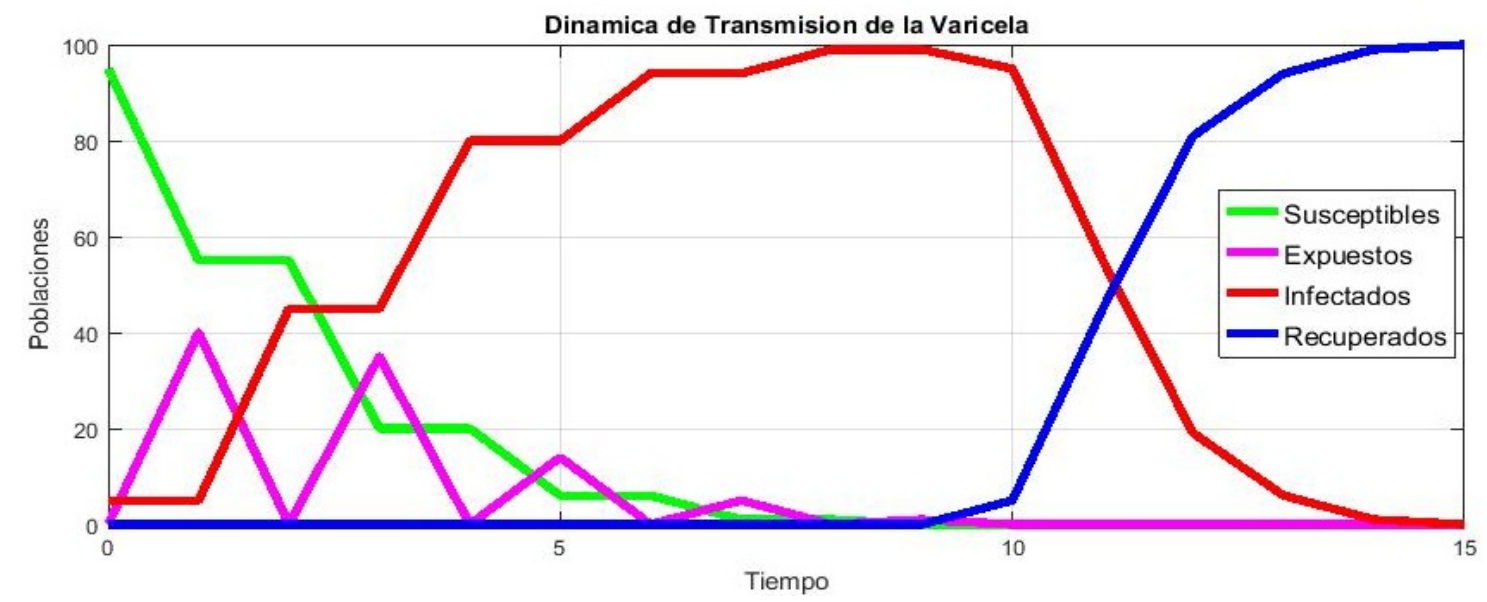

De la secuencia de los estados de las personas en las diversas poblaciones epidemiológicas, se presenta una gráfica similar que se obtuvo mediante el modelo determinístico SEIR en Ecuaciones Diferenciales Ordinarias. De este modelo desarrollado mediante el Tiempo Discreto ha permitido conocer la evolución de la enfermedad, por mientras que el modelo computacional mediante los Autómatas Celulares desarrollado mediante los Eventos Discretos nos permiten tener una concepción similar de la propagación de la enfermedad. [9, 13, 16 y 17].

\section{Conclusiones}

Los Modelos que se han presentado en las Secciones 3 y 4 tanto por las Ecuaciones Diferenciales Ordinarias como también se puede expresar por las Ecuaciones Diferenciales Parciales, pero sobre todo los Autónomas Celulares que permiten conocer la dinámica de transmisión de la varicela, cada uno en su respectiva área. [4, 9, 10 y 16].

En primer lugar, el modelamiento matemático aplicado a la Epidemiología, de manera particular a la Varicela nos permite tener consideraciones básicas para poder describirla y formalizarla en un modelo. Pues en cuanto más detalles y consideraciones tenga será mucho mejor el modelo en cuanto el análisis cualitativo debe garantizar la estabilidad del modelo y complementada por la simulación numérica que se realiza. [1, 2, 4 y 5].

En segundo lugar y la parte fundamental del presente trabajo, es el modelamiento computacional que se puede realizar de la dinámica de transmisión de la Varicela mediante los Autómatas Celulares que nos permiten mediante reglas sencillas poder representar como se genera la evolución de la enfermedad en la población total. Si se tiene en consideración el tamaño la región establecida para la simulación, surge el detalle cual sería el tamaño adecuado para una simulación significativa para una respectiva toma de decisiones para enfrentar la epidemia de la varicela, debido que el costo computacional que se tiene al realizar las operaciones internas del algoritmo computacional. [13, 14 y 16].

Por lo cual, se puede concluir lo siguiente: 
- El modelo computacional mediante Cell-DEVS, nos permite visualizar como se desarrolla la epidemia teniendo en cuenta el tiempo y el espacio de la región considerada para el respectivo análisis epidemiológico.

- La posición de las personas infectadas en la región nos permite analizar en qué lugares se puede generar un brote más rápido, y así poder tener las consideraciones respectivas de prevención y control.

- El formalismo DEVS nos permite definir reglas sencillas de un problema para poder realizar la simulación computacional de tal manera que describa la dinámica del problema. De esta manera, la evolución de la epidemia se puede analizar en tiempo real.

- El adecuado complemento entre la simnulación mediante Métodos Numéricos (Tiempo Discreto o Evento Discreto), y la simulación mediante los Autómatas Celulares permite una visión más amplia de como evoluciona la propagación de la enfermedad en las poblaciones epidemiológicas teniendo en cuenta las ventajas y desventajas de cada método de simulación computacional.

- Los Autómatas Celulares permite la apertura de un camino diferente para generar modelos computacionales que permitan brindar respuestas en tiempo real de acuerdo a las reglas que describen la dinámica de la evolución de la enfermedad.

\section{Referencias Bibliogáficas}

[1] Anderson, R. M. (1982). Population Dynamics of Infectious Diseases: Theory and Applications. Chapman and Hall, London-New York.

[2] Arteaga Bonilla, R., y Arteaga Michel, R. (2004). Infección por Varicela y Vacuna contra Varicela en el Siglo XXI. Revista de la Sociedad Boliviana de Pediatría, 43(3), 181-190.

[3] Arteaga, J. y Sanabria, M. (2014). Modelo de Propagación de la Malaria usando Autómatas Celulares con Indicadores de Probabilidad. Colombia: Universidad Nacional de los Andes.

[4] Brauer, F. y Castillo-Chávez, C. (2001). Mathematical Models in Population Biology and Epidemiology. New York: Springer.

[5] Brisson, M., Edmunds W.J., Gay N.J., Law, B. y De Serres G. (2000). Modelling the impact of immunization on the epidemiology of varicella zoster virus. Epidemiology and Infection, 125, (3), 651-669.

[6] Busenberg, K., Cooke, L. y Pozio M.A. (1983). Analysis of a model of a vertically transmitted disease. Journal of Mathematical Biology, 17(3), 305-329.

[7] Capasso, V. y Serio, G. (1978). A generalization of the Kermack-McKendrick deterministic epidemic model. Mathematical Biosciences, 42(1-2), 43-61.

[8] Dirección General de Epidemiología. (2016). Vigilancia, Prevención y Control de la Varicela. Perú: Ministerio de Salud.

[9] Dirección General de Epidemiología. (2016). Sala situacional para el Análisis de Situación de Salud - SE 30-2017. Perú: Ministerio de Salud.

[10] Elkardry, Alaa. (2013). Transmission Rate in Partial Differential Equation in Epidemic Models. Thesis submitted to the Graduate College of Marshall University, United States. 
[11] Giménez, F. (2011). Vacunación frente a Varicela en el 2011. Recuperado de ibvacunas.com/wp-content/uploads/Varicela-santiago-noviembre-2011.pdf

[12] Kermack, W. y McKendrick, A. (1933). Contributions to the mathematical theory of epidemics-III. Further studies of the problem of endemicity. Proceedings of the Royal Society of London, 141(843), 94-122.

[13] Piqueira, J., De Vasconcelos, A., Gabriel, C. y Araujo, V. (2008). Dynamic models for computer viruses. Computers \& Security, 27(7-8), 355-359.

[14] Toffoli, T. y Margolus, N. (1987). Cellular automata machines: A new environment for modeling. Cambridge, MA: MIT Press.

[15] Trottier, H.y Philippe, P. (2000). Deterministic modeling of infectious diseases: theory and methods. The Internet Journal of Infectious Diseases, 1(2).

[16] Wainer, G. Giambiasi, N. (2002). N-dimensional Cell-DEVS. Discrete Events Systems, $12(2), 135-157$.

[17] Wainer, G. (1998). Discrete-Event cellular models with explicit delays. PhD thesis, Université d'Aix-Marseille III, France. 